\title{
Silicon strip prototypes for the ATLAS Upgrade tracker of the HL-LHC
}

\author{
Sergio Díez, on behalf of the ATLAS Upgrade Collaboration
}

\begin{abstract}
This paper describes the integration structures for the silicon strips tracker of the ATLAS detector for the PhaseII upgrade of the Large Hadron Collider (LHC), also referred to as High-Luminosity LHC (HL-LHC). Silicon strip sensors are arranged in highly modular structures, called 'staves' and 'petals'. This paper focuses on the prototyping effort developed by the strips tracker barrel community, as well as on the description of one of the latest stave prototypes. This new prototype is composed of a particular core structure, in which a shield-less bus tape is embedded in between carbon fiber layups. Electrical and thermal performances of the prototype are presented, as well as a description of the assembly procedures and tools.
\end{abstract}

\section{INTRODUCTION}

The inner tracker of the present ATLAS detector has been designed and developed to function in the environment of the present Large Hadron Collider (LHC). At the next-generation tracking detector proposed for the High Luminosity LHC (HLLHC), the so-called ATLAS Upgrade, the particle densities and radiation levels will be higher by as much as a factor of ten. The new detectors must be faster, more highly segmented, cover more area, be more resistant to radiation, and require much greater power delivery to the front-end systems. At the same time, they cannot introduce excess material which could undermine performance. For those reasons, the inner tracker of the ATLAS detector must be redesigned and rebuilt completely [1].

The design of the ATLAS Upgrade tracker has already been defined. It consists of several layers of silicon particle detectors. The most internal layers will be constituted by silicon pixel sensors, and the external layers will be constituted of silicon "short" $(\sim 2.5 \mathrm{~cm})$ and "long" $(\sim 5 \mathrm{~cm})$ strip sensors. In response to the needs of the strip region for the upgraded tracker, highly modular structures are being studied and developed, called "staves" for the central region (barrel) and "petals" for the forward regions (end-caps). These structures integrate large numbers of sensors and readout electronics, with precision light weight mechanical elements and cooling structures. In the case of the barrel region, the baseline design of a stave consists of single-sided silicon strip sensors, glued onto laminated kapton-copper bus tapes at the

Manuscript received November 16, 2012. This work was supported in part by the Director, Office of Science, Office of Basic Energy Sciences, of the U.S. Department of Energy under Contract No. DE-AC02-05CH11231.

Full names of authors are preferred in the author field, but are not required. Put a space between authors' initials. Do not use all uppercase for authors' surnames.

Sergio Díez is with the Lawrence Berkeley National Laboratory, Berkeley, CA 94720 USA (telephone: 510-495-2461, e-mail: sdiezcornell@lbl.gov). two sides of a low mass carbon-based core structure with embedded titanium cooling pipes. Readout, control, and power electronics are hosted in kapton flex hybrids, glued directly to the silicon sensors with electronic grade epoxy. A stave hosts 13 identical sensor modules per side, having both axial and stereo strips providing 3D coverage. The petals for the forward regions follow closely the design of the staves; they are not covered in this paper. The development of the stave and petal prototypes is an effort driven by a collaboration of more than 20 institutes worldwide [2]. This paper briefly summarizes some of the latest progress of this collaboration and describes in detail one of the latest stave prototypes recently assembled. An alternative integration concept for the ATLAS Upgrade strips tracker is the so-called 'supermodule' concept. Detailed studies for this approach are published elsewhere [3].

\section{STRIPS TRACKER BARREL PROTOTYPES}

A stave in the strips barrel hosts 13 modules per side, 26 in total. The increased number of channels and hence the higher dissipated power of the short strips modules with respect to the long strips modules makes them the most challenging. For that reason, the barrel community quickly focused the prototyping efforts on the short strip region.

\section{A. Short strips modules}

The silicon microstrip sensors are fabricated in n-in-p float zone (FZ) technology, consisting of a segmented $n^{+}$implant on a p-bulk technology. This technology exhibits numerous advantages: it collects electrons, which makes it faster than current ATLAS p-in-n microstrips; it depletes from the segmented side, achieving good signal even under-depleted; and finally, it is a single-sided process, making it cheaper than double-sided n-in-n technology, with more foundries and available capacities worldwide. The ATLAS collaboration, in collaboration with Hamamatsu Photonics, Ltd., has developed $9.75 \times 9.75 \mathrm{~cm}^{2}$ micro-strip sensors for the short strips region of the tracker, segmented in 4 columns of 1280 strips. Two of those columns include axial strips, while the other two include stereo strips with a $40 \mathrm{mrad}$ angle. Each strip is $2.39 \mathrm{~cm}$ long, and the pitch between strips is equal to $74.5 \mu \mathrm{m}$. Each short strip sensor has in total 5120 strips, and the devices are $310 \mu \mathrm{m}$ thick. More details about the sensor design and electrical characterization can be found in [4], [5].

Each short strip module includes two flex hybrids, hosting the readout electronics and passive components necessary for the readout of the 5120 channels, plus part of the power 
circuitry. Current prototypes use the $\mathrm{ABCN}-25$ binary readout ASICs, fabricated on $0.25 \mu \mathrm{m}$ CMOS technology, each with 128 channels. $40 \mathrm{ABCN}-25$ ASICs are required for the readout of a short strips module, 20 on each hybrid. Each hybrid requires one Basic Control Chip (BCCs) ASIC, also fabricated on $0.25 \mu \mathrm{m}$ CMOS technology, which in the current prototypes is located on a daughter Printed Circuit Board (PCB) next to the hybrid, connected to it via wire-bonds. The final version of the short strip modules will include the $0.13 \mu \mathrm{m}$ CMOS version of the readout electronics (the ABCN13 ASIC), each with 256 channels. This ASIC is currently in its final stage of design. In this case, only $10 \mathrm{ABCN}-13$ will be required per hybrid. In addition, the BCCs will be also replaced by the $0.13 \mu \mathrm{m}$ CMOS Hybrid Control Chip (HCC). One HCC per hybrid will be required, and will be hosted in the hybrids.

Two power architectures have been considered for the staves: serial powering and DC-DC powering. In serial powering, a constant current source provides power to all the modules of a stave side with a shunt regulator circuit and a single power line. The current is constant along the chain, and is equal to the current required by an individual hybrid or module. There are as many voltage steps as elements in the chain, and protection circuitry is required to prevent open circuits in the serial power line [6]. Another approach is used in DC-DC powering, in which a constant voltage source provides power to the modules in a stave side after one or several voltage conversion steps made by buck DCDC converters. It also requires a single power line. Current increases along the stave, and the total current at the end of the stave is equal to the number of modules in the stave multiplied by the current required per hybrid and divided by the voltage conversion ratio [7]. Strong efforts have been dedicated by the collaboration to the study and development of both powering architectures, each with advantages and drawbacks, and both powering architectures have been extensively tested with short strip module and stave prototypes [8]. More details about both power distributions can be found in [9].

Almost 21000 strip modules need be installed in the upgraded ATLAS tracker, 13000 of them in the barrel region. For that reason, module production has been planned with large scale requirements even at a prototyping stage. The flex hybrids are fabricated at Stevenage Circuits, Ltd. (UK), following a panelized design, to allow machine placement of passive components and solder reflow. Fig. 1 shows one of these panels. Each panel hosts 8 hybrids. Diverse tools have been developed for the controlled gluing and wire bonding of the ABCN ASICs to the panelized hybrids. Conductive TRADUCT 2902 epoxy was selected for the gluing of the ASICs onto the hybrid panels. Electrical tests can be performed on the fully populated hybrids. Once the hybrids are tested, they are removed from the panels and glued directly onto the silicon strip sensors with semiconductor grade Epolite FH5313. This glue has been extensively tested on miniature microstrip sensors and under proton irradiations at fluences up to $1.5 \times 10^{15} n_{e q} / \mathrm{cm}^{2}$. Sensors held breakdown voltages well over $600 V$ [2]. Glue thickness and uniformity is controlled with precision mechanical tools, and an optimized glue thickness of $\sim 120 \mu \mathrm{m}$ is achieved [10]. Automated wire-bonding procedures to bond two rows of 64 channels per ASIC (128 channels in total) from the ABCN-25 to the sensor microstrips have also been developed at the different construction sites. The stave modules are finally placed in removable PCB frames that provide the traces and connections between the hybrids, power circuitry and DAQ system, and the placement and connection of the BCC boards. These low cost frames allow the electrical test of individual modules with different powering distributions, grounding and shielding configurations, added circuitry, etc. Fig. 2 shows a short strip module fully assembled and ready for test. More than 160 hybrids and 62 modules have been assembled and tested with high yield by the different institutes within the collaboration. More details about electrical testing of individual hybrids and modules before and after irradiation can be found in [2], [10], [11].

\section{B. Stave prototypes}

3 small prototype versions of the barrel short strips staves have been also manufactured following the baseline design. The so-called 'stavelets' consist of of single-sided stave prototypes with 4 strip modules directly glued onto the bus tape, which includes the copper trace layer under an aluminum shield and several Kapton and adhesive layers. The bus tapes are co-cured with the carbon fiber (CF) facings and laminated on both sides of the stavelet carbon core structure. This cocuring process is performed under a profiled surface in order to correct deformations which appear due to a mismatch of the coefficients of thermal expansion (CTE) between the aluminum and the CF. The edges of the tapes have been widened to provide space to attach the power and protection components on one edge, and the $\mathrm{BCC}$ boards on the other. The stavelet prototypes have proved to be very powerful test beds for electrical testing, such as the different powering distributions, grounding and shielding configurations, etc. Apart from a shield-less stavelet with a modified layout, which will be discussed in section III, three additional stavelets have been built so far following the baseline layout: two of them with serial powering, and one with DC-DC powering. Similar electrical behavior has been observed for all three stavelets: an overall input noise increase of $\sim 20 e^{-}$for threshold scans at $1 f C$ injected charge is observed with respect to the same individual modules mounted on test frames, prior

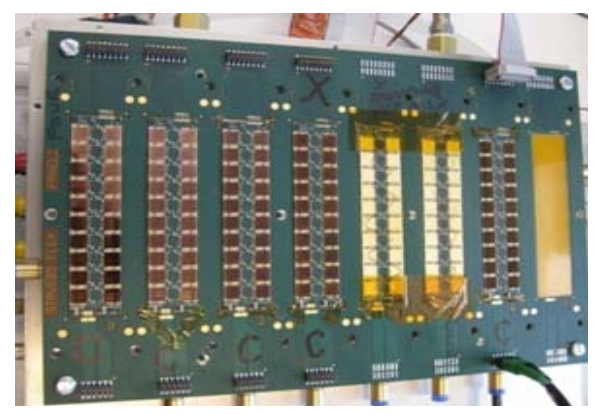

Fig. 1. Hybrid panel, with 5 of its hybrids populated with ASICs. 


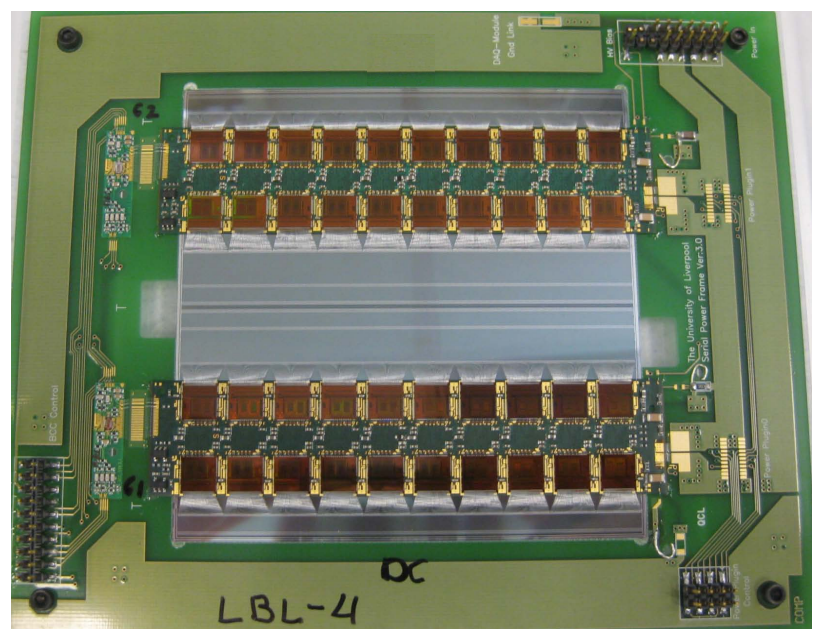

Fig. 2. Short strips module. The module is placed on a serial power frame. BCC boards can be seen at left side of the hybrids.

to be mounted on the stavelets. A more detailed description of the baseline stavelets, and an in-depth discussion of the electrical results obtained from these prototypes can be found in [8], [12]. A full-size stave prototype (12 modules per side) is currently under construction.

\section{SHIELD-LESS STAVELET}

A fourth stavelet prototype has been recently fabricated with some unique features. In this prototype, the baseline design of the bus tapes is modified: the $50.8 \mu \mathrm{m}$ thick aluminum shielding layer is completely removed all along the sensor backplane, leaving only a thin, $\sim 6 \mathrm{~mm}$ wide shielding trace above the LVDS control lines. The CF facings act as an effective shielding for the data lines, which run below the sensor. In addition, this prototype is the first stavelet meant to be double-sided, although at the moment only one of the sides has been populated with modules. The motivation for this modified layout comes from several aspects: first, the removal of the aluminum shielding layer will imply a $50 \%$ material reduction in the tapes, leading to an overall $10 \%$ reduction of the stave radiation length, one of the most critical parameters of the tracker components. Second, the complexity of the tape manufacture and co-curing is greatly reduced, which in turn leads to a cost reduction of the tapes; this is related to the high mass and thickness of the Al layer $(50.8 \mu \mathrm{m}$ thick as opposed to, for example, the layer with the $\mathrm{Cu}$ traces, $17.8 \mu \mathrm{m}$ thick, and only with a few traces along the stave area) and to differences among the CTEs of the different tape and facings layers ( $\mathrm{CF}$, Kapton, $\mathrm{Cu}, \mathrm{Al}$, adhesives). The different CTE values lead to measurable deformations during the tape manufacture, and especially during its co-curing along with the facings. These deformations have to be corrected with mitigation techniques. Once the Al layer is mostly removed, deformations become marginal. Finally, the removal of an additional, thick layer between the cooling pipes and the silicon sensors may enhance the thermal performances of the stavelet prototype.

\section{A. Shield-less stavelet core}

Similarly as the baseline layout of the staves, the core consists of a 'honeycomb' CF body with an embedded, Ushaped stainless steel cooling pipe, wrapped in high thermally conductive carbon foam. The Outer Diameter (OD) of the pipe is equal to $3.3 \mathrm{~mm}$, and the wall thickness is $220 \mu \mathrm{m}$. The final version of the stave is envisioned to have a titanium cooling pipe with $2.4 \mathrm{~mm}$ OD and $140 \mu \mathrm{m}$ thick walls. The core structure is completed with the laminated multilayer 0-90-0 CF facings (the numbers account for the different orientations of the fibers of each lay-up), co-cured together with the bus tapes. In the baseline stave design, briefly discussed in section II-B, the tape is co-cured on top of the CF skins. In this particular case, however, the tapes are co-cured in between two lay-ups, and the last CF skin, with its size reduced along the edges, acts as an effective shielding layer. Fig. 3 shows both the baseline and modified designs of the bus tape. The Al shielding layer has been kept at the location of one of the modules in this prototype, in order to compare both layouts. The lateral close-outs of the core are also made out of $\mathrm{CF}$ composites. The total thickness of the core structure is equal to $5 \mathrm{~mm}$. The modules are directly glued onto the last $\mathrm{CF}$ layer with thermally conductive SE4445 epoxy from Dow Corning. The backplane contact of the silicon sensors is made with electrically conductive TRA-DUCT 2902 epoxy at the HV pads of the tape. An additional Kapton layer (Kapton tape) is required between the $\mathrm{CF}$ and the conductive epoxy at the vicinity of the HV pads, in order to avoid the creation a shortcircuit between the sensors backplane and the CF shield layer.
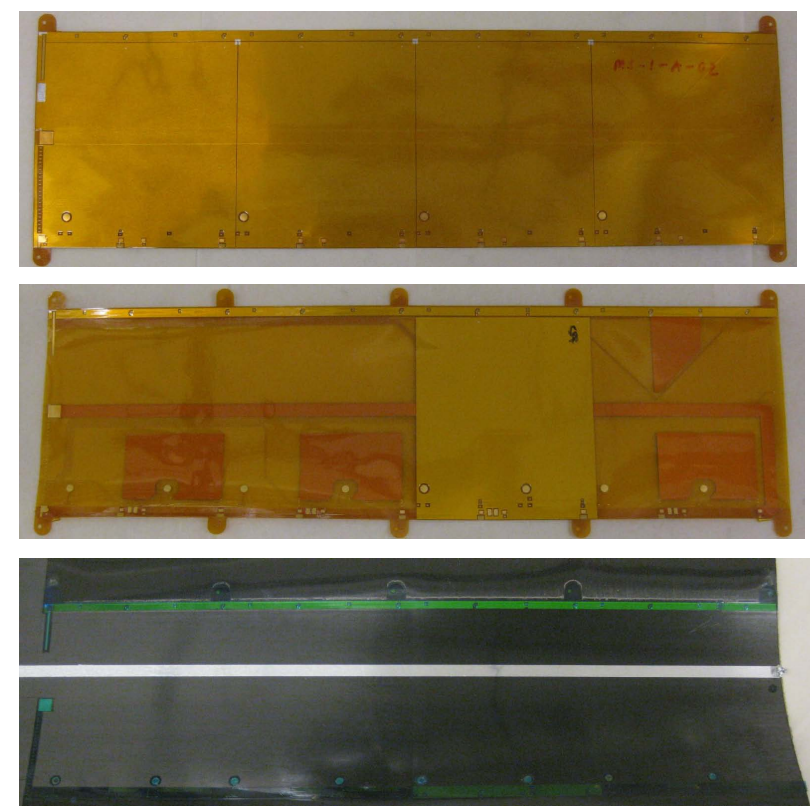

Fig. 3. (Top) Baseline layout bus tape, with Al shielding covering all the area below the silicon detectors. (Middle) Modified, shield-less bus tape, with Al shielding only on top of the LVDS control traces, on the upper edge of the tape. The Al shielding is left at one module location for comparison. (Bottom) Modified bus tape co-cured in between two lay-ups of CF and before lamination into the stavelet core. Pads are covered with low tack blue tape for protection during co-curing. 

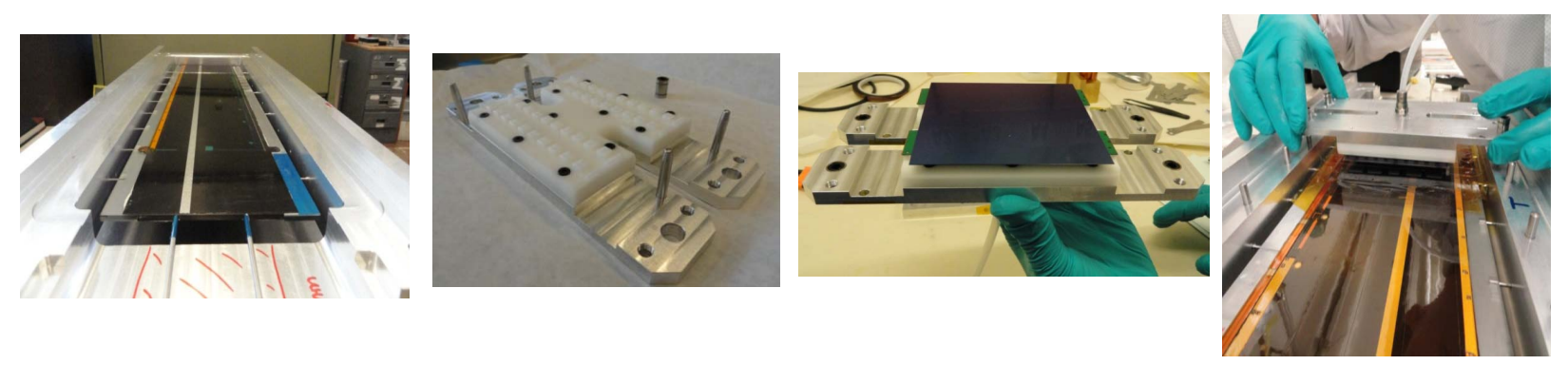

Fig. 4. Stavelet assembly tools. From left to right: frame with stavelet attached; pickup tool; module held by the pickup tool; assembly of a stavelet module.

\section{B. Stavelet assembly and components}

Precision mechanical tools were designed in order to glue the modules onto the stavelet core with the required precision. The tools design is strongly based on the short strips module mounting tools. A custom design pickup tool holds under vacuum the fully assembled and wire-bonded modules from the ASICs surface. The core is attached from its lateral closeouts during assembly in a custom stavelet frame by means of $2 \mathrm{~mm}$ diameter flip pins. The pins are placed in position with set screws from the outer edges of the frame. The frame also includes vertical $5 \mathrm{~mm}$ diameter press pins which, along with 4 linear bearings on the pickup tool, determine the position of the modules with the required accuracy. A glue mask with a well defined pattern, also based on the one used for the baseline stavelets, determines the glue pattern on the sensor backplane. The glue mask is designed to maximize the SE4445 glue spread and hence the thermal conduction between the stavelet core and the strips modules. The glue thickness is controlled with the linear bearings on the pickup tool. These tools allow a uniform, $\sim 175 \mu \mathrm{m}$ glue thickness, and a module position accuracy within $\sim 150 \mu \mathrm{m}$ for the $X$ and $Y$ dimensions, well above the minimum precision required for this prototype $(500 \mu \mathrm{m})$. Fig. 4 shows these tools during the assembly of a module.

Besides the modules themselves, additional readout, control and power components are required: BCC ASICs/boards (one per hybrid), buck DC-DC converters (one per hybrid) connected in a 'star' topology to the power lines, and the End of Stave (EOS) board. The EOS board includes the MLVDS drivers for the command lines, individual AC-coupled receivers for the data lines coming from each hybrid, additional temperature digitization by means of Analogue-toDigital Converters (ADCs), Low Voltage (LV) and High Voltage (HV) power pass through, and high density voltage and current connectors. Fig. 5 shows the shield-less stavelet completely assembled and ready for test.

\section{Test setup}

Electrical test of hybrids and modules requires dealing with highly multiplexed signals at high frequencies. The baseline DAQ system designed for that purpose is the High Speed Input/Output (HSIO) board [13]. It consists of a generic DAQ board with a single Virtex-4 Field Programmable Gate Array (FPGA) that allows data processing and connection to a controller PC. Additionally, the BCCs provide AC-coupled Low Voltage Differential Signals (LVDS) clock and command, and generate the $80 \mathrm{MHz}$ clock from the common $40 \mathrm{M} \mathrm{Hz}$ LVDS clock. Up to 64 simultaneous data streams are supported with this system. The system also uses an upgraded version of the sctdaq software, used in the past for the test of the current Semiconductor Tracker (SCT) modules [14]. The HSIO is connected to the EOS on the stavelet with SAMTEC ribbon cables. During operation, the stavelet is kept inside a test box to shield the sensors from light. The stavelet runs cooled down by means of a Neslab CFT-33 water chiller, operating at $6^{\circ} C$. The test box is flooded with nitrogen to prevent moisture on the stavelet. Custom VME power supplies are used for the HV of the sensors, running at $200 \mathrm{~V}$. LV power for the DC-DC converters, running at $10.5 \mathrm{~V}$, is provided by a Sorensen XPF 60-20D test bench power supply.

\section{Electrical performances}

The test setup described in the previous section allows the standard threshold scans for binary readout systems. One of the typical figures of merit of these tests is the input noise for each readout channel. It is obtained from the occupancy curves ('s-curves') of each channel at a particular injected charge. The $50 \%$ occupancy point is extracted from the s-curve, along with the output noise (the width of the s-curve). The response curve can then be obtained ( $50 \%$ occupancy point vs. injected charge). The gain of the channel is calculated as the derivative of the response curve. Finally, the input noise or Equivalent Noise Charge (ENC) noise is calculated as the gain divided by the output noise (represented in $e^{-}$). A typical target value for the stavelet prototypes is a signal-to-noise ratio $S / N \geq 10$ at $1 \mathrm{fC}$ injected charge, which turns into $600-650 e^{-}$. Table I shows the average ENC noise at $1 f C$ injected charge for each of the chip columns of the shield-less stavelet. A clear columnto-column noise variation can be observed on the results shown in table I. This variation is consistent with the fact that, within each module, the outer strip columns are mostly covered by the hybrids, while the inner strips are mostly uncovered. For that reason, the readout ASICs of the outer columns ( $\mathrm{C} 0$ and C3) see an extra capacitive load due to the capacitance between the strips and the shield layer of the hybrids. This extra capacitance depends on the hybrid shielding layer and the glue thickness between the hybrid and the sensor. Another interesting result is that the $\mathrm{Al}$ shielded module (module 1) does not exhibit ENC better noise performances than the three 


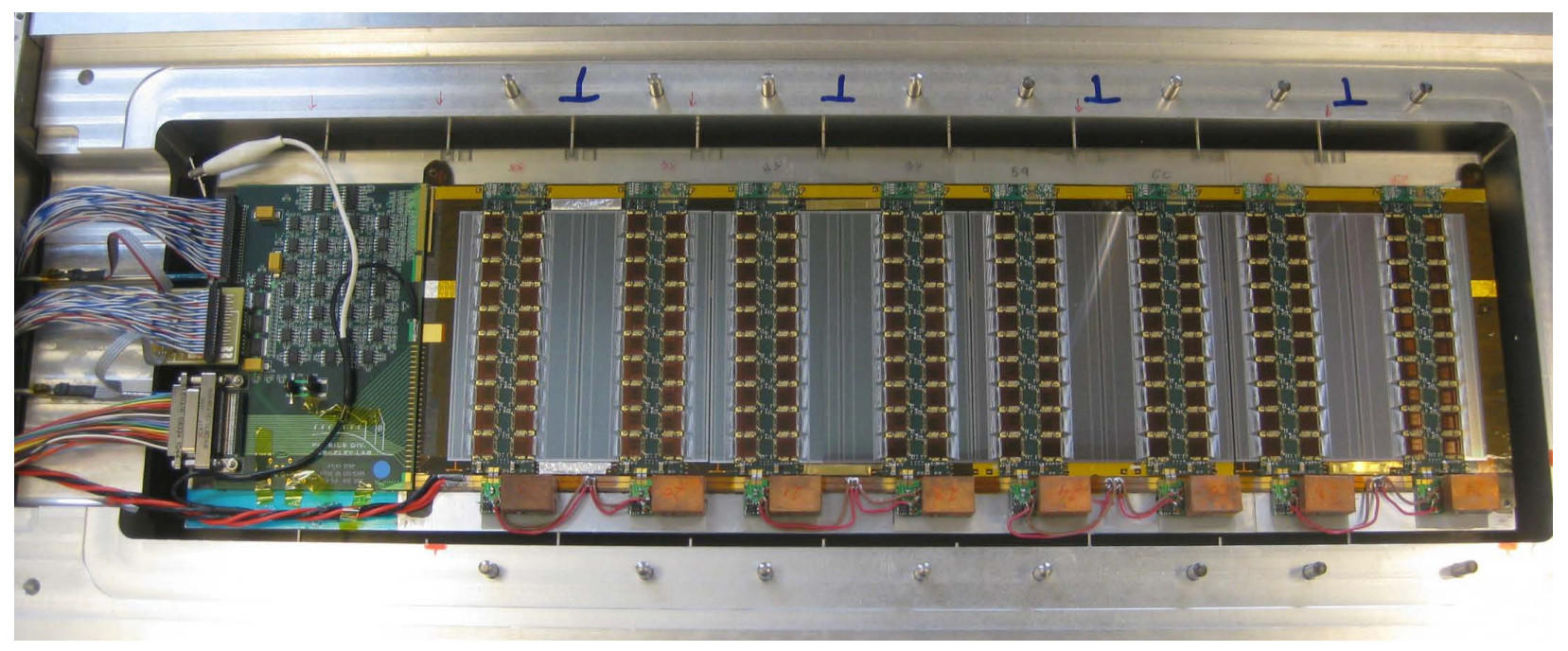

Fig. 5. Shield-less stavelet assembled and ready for test. BCC boards can be seen on top, DC-DC converters at the bottom, and the EOS board on the left.

TABLE I

ELECTRICAL PERFORMANCES OF THE SHIELD-LESS STAVELET

\begin{tabular}{|c|c|c|c|c|c|}
\hline Module & Column & ENC $\left(e^{-}\right)$ & DTN $(1 f C)$ & DTN $(0.75 f C)$ & DTN $(0.5 f C)$ \\
\hline \multirow{3}{*}{ Module 3 } & C0 & 637 & 0 & 0 & 5 \\
& C1 & 635 & 0 & 0 & 31 \\
& C2 & 625 & 0 & 0 & 12 \\
& C3 & 642 & 0 & 0 & 2 \\
\hline \multirow{3}{*}{ Module 2 } & C0 & 680 & 0 & 0 & 2 \\
& C1 & 659 & 0 & 0 & 7 \\
& C2 & 628 & 0 & 0 & 9 \\
& C3 & 647 & 0 & 0 & 2 \\
Module 1 & C0 & 649 & 0 & 0 & 6 \\
(Al shielded) & C1 & 625 & 0 & 0 & 16 \\
& C2 & 616 & 0 & 0 & 8 \\
\hline \multirow{3}{*}{ Module 0 0} & C0 & 645 & 0 & 0 & 5 \\
& C1 & 634 & 0 & 0 & 14 \\
& C2 & 641 & 0 & 0 & 4 \\
\hline
\end{tabular}

CF-shielded modules. Finally, results observed in the shieldless stavelet are completely comparable to the ones obtained with the baseline DC-DC stavelets: in that case, ENC noise at $1 \mathrm{fC}$ remained between 612 and $689 e^{-}$for all hybrids [8].

During a threshold scan, occupancy data is never recorded during data transfer. In the real case, however, the stave is a 'deadtimeless' system: that is, charge integration will be performed at the same time as the data readout. A well known issue for charge measurement systems is that readout trigger signals may produce noise interference during simultaneous charge integration. In order to probe that effect, the so-called double trigger noise test was implemented on sctdaq. It consists of the following: for a particular set of fixed thresholds, a first trigger signal is sent to the readout electronics. Data read out obtained with that trigger is discarded. Then, after a controlled number of clock cycles, equal or close to the length of the cell pipeline of the readout ASICs, a second trigger is sent. The readout data obtained with the second trigger is looking at the charge integration occurred exactly at the same time as the first trigger signal. This will then probe the effect of the first trigger to the charge collection.
Since no charge is injected, ideally the number of hits read out at that particular time stamp should be zero. Table I also shows the number of hits produced in each hybrid column after a double trigger noise test, for a time interval of around 20 clock cycles, centered in the ABCN-25 pipeline (132 cells), for three different fixed threshold values (corresponding to an injected charge equal to $0.5,0.75$, and $1 f C$ ). Results are completely clean at the two higher threshold values in all cases, and remain below 35 hits in all cases at the lowest threshold value. This little extra occupancy does not interfere with the normal operation of the modules. Again, there are not significant differences between the Al shielded module and the $\mathrm{CF}$ shielded modules. This encouraging result is also comparable to the one observed at the baseline DC-DC stavelet prototype, in which the number of hits remained below 40 even at the lowest threshold [8].

\section{E. Thermal performances}

Each hybrid hosts a Negative Temperature Coefficient (NTC) thermistor in its center. In addition, SHT71 Relative Humidity $(\mathrm{RH})$ and temperature sensors are located at the inlet 
TABLE II

THERMAL PERFORMANCES OF THE SHIELD-LESS STAVELET

\begin{tabular}{|c|c|c|c|c|c|c|c|c|c|}
\hline \multicolumn{2}{|c|}{ Cooling pipe } & \multicolumn{2}{|c|}{ Module 3 } & \multicolumn{2}{c|}{ Module 2 } & \multicolumn{2}{c|}{ Module 1 } & \multicolumn{2}{c|}{ Module 0 } \\
\hline Inlet & Outlet & Hybrid 0 & Hybrid 1 & Hybrid 0 & Hybrid 1 & Hybrid 0 & Hybrid 1 & Hybrid 0 & Hybrid 1 \\
11.9 & 12.7 & 23.6 & 27.5 & 23.6 & 23.4 & 26.1 & 25.3 & 24.6 & 21.0 \\
\hline
\end{tabular}

and outlet of the cooling pipes right after the stavelet core. The read out of both the SHT71 and NTCs is implemented in the sctdaq software. This allows the study of the thermal performances of the stavelet during operation. Table II shows the temperature values observed at the different hybrids and at the inlet and outlet of the cooling pipe. In addition, Fig. 6 shows an infrared image of the stavelet during operation, taken with a FLUKE Flexcam. From the image it can be clearly observed that the biggest heat sources of the system are the DC-DC converters. From the temperature values coming from the NTCs, it can also be inferred that the region of the $\mathrm{Al}$ shielded module exhibits slightly higher temperatures than the other three modules, with the exception of one of the hybrids on module 3 . As expected, this seems to indicate that the region without the $\mathrm{Al}$ shielding exhibits better thermal performances than the $\mathrm{Al}$ shielded region, just by removing the additional thick Al layer between the module and the cooling pipes. Although differences are small in this case, it might become a significant difference in a stavelet with the full Al shield layer. On the other hand, the slight $\mathrm{T}$ increase in the shield-less stavelet is not sufficient to translate into a measurable ENC noise increase. The small difference between inlet and outlet pipes $\left(\Delta T \leq 1^{\circ} C\right)$ indicates again the excellent thermal performances of the stavelet. Unfortunately, a direct comparison with the other stavelet prototypes is not straightforward in this case, given the significant differences in the test setup for the different prototypes.

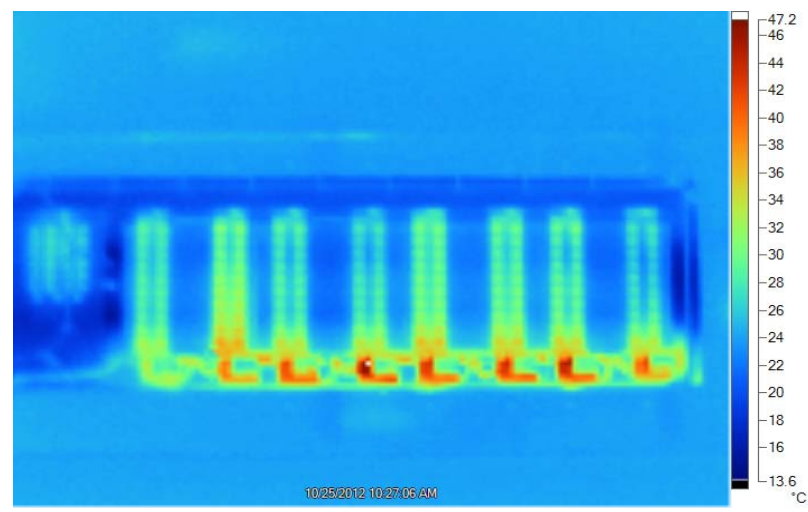

Fig. 6. Infrared image of the shield-less stavelet during operation.

\section{CONCLUSION}

The stave program for the Phase-II ATLAS Upgrade has shown significant progress recently. More than 60 short strip module prototypes, with both powering schemes implemented, have been built and shown to work so far, even after irradiation at higher fluences than expected on the Si tracker. Numerous construction sites within the collaboration have joined the effort, involving more than 20 institutes. 4 single-sided stavelets have been also fabricated, and more prototypes are underway. In particular, a low-mass shield-less stavelet has been recently assembled at LBNL. New core assembly techniques have been developed, along with new cost-effective mechanical tools for its assembly. Similar electrical performances as previous shielded prototypes have been demonstrated, both for the shielded and shield-less modules. In addition, excellent thermal performances have been observed, in particular at the shield-less modules. These results suggest that the removal of the shielding layer on the bus tapes does not undermine the performances of the stave prototypes, although this results have to be confirmed once the backside of the shield-less stavelet is populated. Next generation stave prototypes are currently under design in the collaboration, and will be built as soon as the ABCN-130 and HCC ASICs are ready. The fabrication of the first ASICs is expected in approximately 6 months from now.

\section{ACKNOWLEDGMENT}

The authors would like to thank the undergraduate students A. Faroni and T. Txiao, from University of California Berkeley (USA), and M. Defferrard, from University of Fribourg (Switzerland) for his dedication and efficient work at Berkeley Lab during the different stages of the project.

\section{REFERENCES}

[1] F. Gianotti, M. Mangano and T. Virdee (conveners), "Physics potential and experimental challenges of the LHC luminosity upgrade," Eur. Phys. J. C, vol. 39, no. 3, pp. 293-333, 2005.

[2] P.P. Allport et al., "Progress with the single-sided module prototypes for the ATLAS tracker upgrade stave," Nucl. Inst. Meth. A, vol. 636, no. 1, Supplement 1, pp. S90 - S96, 2011.

[3] S. Gonzalez-Sevilla et al., "Electrical performance of a silicon micro-strip super-module prototype for the High-Luminosity LHC collider," Nucl. Inst. Meth. A, 2012. [Online]. Available: http://www.sciencedirect.com/science/article/pii/S0168900212003063

[4] Y. Unno et al., "Development of n-on-p silicon sensors for very high radiation environments," Nucl. Inst. Meth. A, vol. 636, no. 1, Supplement, pp. S24 - S30, 2011.

[5] J. Bohm et al., "Evaluation of the bulk and strip characteristics of large area n-in-p silicon sensors intended for a very high radiation environment," Nucl. Inst. Meth. A, vol. 636, no. 1, Supplement, pp. S104 - S110, 2011.

[6] D. Lynn et al., "Serial power protection for ATLAS silicon strip staves," Nucl. Instrum. Methods Phys. Res., Sect. A, vol. 633, no. 1, pp. $51-$ 60, 2011.

[7] F. Faccio et al., "Development of custom radiation-tolerant DCDC converter ASICs," J. Inst., vol. 5, no. 11, p. C11016, 2010.

[8] P. W. Phillips, "ATLAS strip tracker stavelets," J. Inst., vol. 7, no. 02, p. C02028, 2012.

[9] S. Diez, "System Implications of the Different Powering Distributions for the ATLAS Upgrade Strips Tracker," Phys. Proc., vol. 37, pp. 960 - 969, 2012.

[10] A. Affolder, "Silicon Strip Detectors for the ATLAS HL-LHC Upgrade," Physics Procedia, vol. 37, pp. 915 - 922, 2012.

[11] S. Diez, on behalf of the ATLAS Collaboration, "Silicon strip staves and petals for the ATLAS Upgrade tracker of the HL-LHC," Nucl. Inst. Meth. A, 2012. [Online]. Available: http://www.sciencedirect.com/science/article/pii/S0168900212006407 
[12] J. Matheson, "Progress and advances in Serial Powering of silicon modules for the ATLAS Tracker Upgrade," J. Inst., vol. 6, no. 01, p. C01019, 2011.

[13] D. Nelson. (2010) HSIO Development Users guide. [Online]. Available: www.slac.stanford.edu/ djn/Atlas/hsio/

[14] L. Eklund et al. (2002) ATLAS SCT Test DAQ Online Documentation. [Online]. Available: http://sct-testdaq.home.cern.ch/scttestdaq/sctdaq/sctdaq.html 